\title{
A General Tactile Approach for Grasping Unknown Objects with a Humanoid Robot
}

\author{
Philipp Mittendorfer, Eichii Yoshida, Thomas Moulard and Gordon Cheng
}

\begin{abstract}
In this paper, we present a tactile approach to grasp large and unknown objects, which can not be easily manipulated with a single end-effector or two-handed grasps, with the whole upper body of a humanoid robot. Instead of conventional joint level force sensing, we equip the robot with various patches of HEX-o-SKIN - a self-organizing, multi-modal cellular artificial skin. Low-level controllers, one allocated to each sensor cell, utilize a self-explored inverted jacobian-like sensory-motor map to directly transfer tactile stimulation into reactive arm motions, altering basic grasping trajectories to the need of the current object. A high-level state machine guides those low-level controllers during the different states of the grasping action. Desired contact points, and key poses for the trajectory generation, are taught through forceless tactile stimulation. First experiments on a position controlled robot, an HRP-2 humanoid, demonstrate the feasibility of our approach. Our paper contributes to the first realization of a self-organizing tactile sensor-behavior mapping on a full-sized humanoid robot, which enables: 1) a new general approach for grasping unknown objects with the whole-body; and 2) a novel way of teaching behaviors using pre-contact tactile sensing.
\end{abstract}

\section{INTRODUCTION}

1) Motivation: Although a growing set of every day objects can be potentially manipulated with common endeffectors, there will always remain a large class of objects, which can not be dealt with - e.g. due to size, weight, the lack of stable grasping points or precise object models. Still being able to efficiently grasp and hold those objects will have a large impact in households, care giving or industrial scenarios - robots could e.g. help to (un-)load airplanes, handle bags of clothes in an industrial laundry or deliver parcels in an office. For such tasks multi-modal, large-area surface sensation seems predestined, as it provides a rich and direct feedback from numerous simultaneous contact points and from a potentially large area of contact. Programming task and robot knowledge excludes non-specialists, is error prone and cumbersome. We were thus motivated to let the robot autonomously explore its configuration and teach the task related knowledge through direct physical interaction.

2) Related Works: Common end-effector manipulations, like in [1], imply a nearly perfect knowledge of the object, the existence of suitable grasping points and a robot with enough power along the entire kinematic chain. Providing tactile sensors, like in [2], the required object knowledge can be relieved - the grasp is becoming reactive [3]. As

P. Mittendorfer and G. Cheng are with the Institute for Cognitive Systems, Technische Universität München, Munich, Germany Email: see http://www.ics.ei.tum.de E.Yoshida and T. Moulard are with the CNRS-AIST JRL (Joint Robotics Laboratory), UMI3218/CRT, Tsukuba, Japan Email: see https://jrlserver.muse.aist.go.jp/

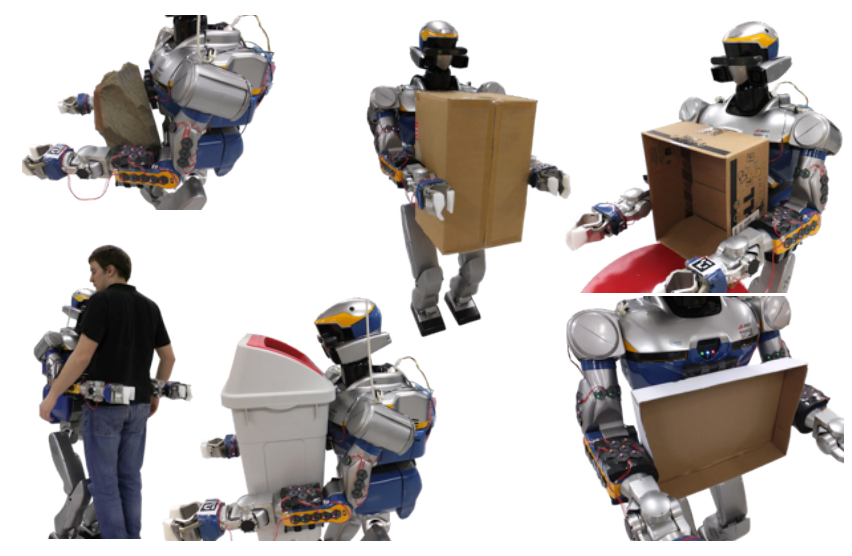

Fig. 1. A position controlled HRP-2 humanoid, holding unknown objects with the whole body, as result of a multi-modal tactile grasping sequence.

demonstrated in [2], the grasping sequence can be split into discrete states with different sets of control parameters. In contrary to control strategies, which we wish to extend from manipulators to the whole body [4], we do not wish to lose the controllability in the upper body of a humanoid robot, excluding passive compliance as an option. Joint level force sensing enables computed compliance [5], but in case of an inaccurate kinematic/dynamic model or a multi-contact scenario, joint level force sensing quickly reaches its limit as: (i) forces sum up to zero; (ii) it is not possible to tell internal from external forces; (iii) variable levers prevent magnitude measurements. Artificial skin can fill this gap, providing a rich and direct feedback, but has received little attention yet. In [6] tactile sensors are utilized to control the contact between a human-like object and the arms of a nursing robot. The approach is currently limited to fine manipulation around an initial contact state. In [7] tactile feedback and additional contact points enable a humanoid to lift heavy objects. Alas, the paper is not very precise on the haptic control strategy - we estimate tactile feedback solely serves to switch between pre-computed procedures. In this paper, we utilize the second generation of our multi-modal sensors [8], which we first introduced in [9]. Previously published self-organization algorithms, like the structural exploration [10] and the generation of the sensory-motor map [11], have been fused. The HRP-2 [12] sub-joint space control has been implemented with a generalized inverted kinematics - the stack of tasks (SoT) [13].

3) Contribution: For the first time, we apply our multimodal artificial skin, and its self-organizing features, on a full-sized humanoid robot. A general tactile approach for 
grasping unknown objects is introduced, which efficiently takes advantage of a distributed, multi-modal sense of touch. In comparison to existing approaches, our novel grasping algorithm requires little knowledge on the robot it controls (no kinematic/dynamic model) and the object it handles (no object model). Utilizing pre-contact sensors for a novel way of teaching behaviors through direct tactile interaction, it is not necessary to apply force on the robot or even touch it - making heavy or position controlled robots featherlight to interact with. Relying on artificial skin, no joint level force sensing is required. Our approach provides a new and complementary level of direct physical interaction.

\section{SYSTEM DESCRIPTION}

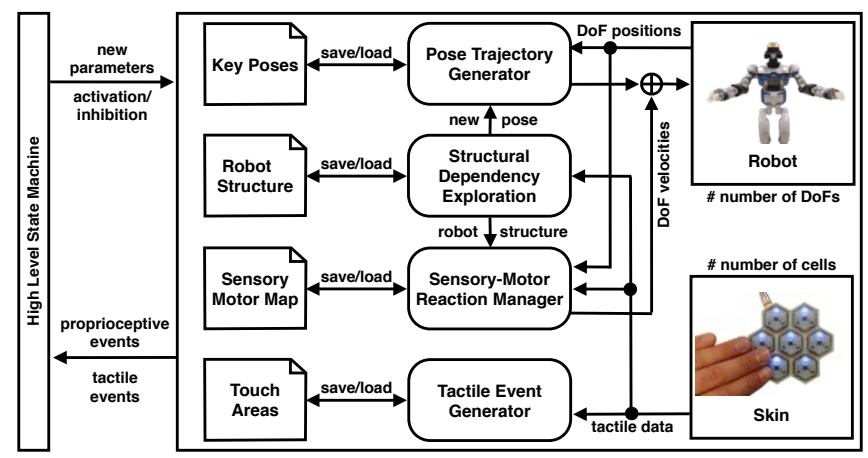

Fig. 2. System diagram: Showing the data exchange between the robot, the artificial skin, the long term memory and the controller sub-blocks. The state machine controls sub-block activity and parameter distribution.

In this section, we introduce the artificial skin system and describe the control interface to the humanoid robot.

\section{A. Artificial Skin}
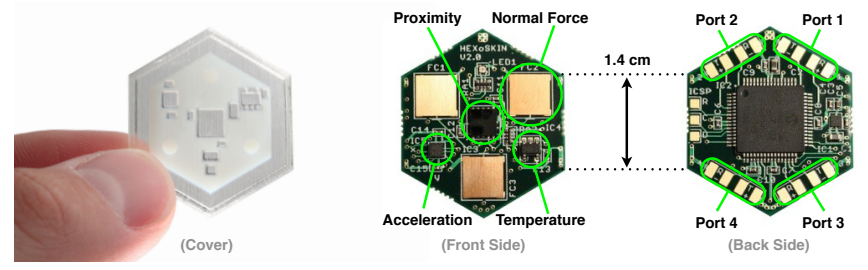

Fig. 3. HEX-o-SKIN unit cell. Front side with 4 sensor modalities. Back side with micro controller and 4 power/data ports. The micro-structured composite cover supports and protects all four embedded sensor modalities.

Our artificial skin (HEX-o-SKIN) builds from rigid, hexagonally shaped sensor cells (SCs) (see Fig. 3). Multiple SCs are directly placed next to each other into elastomer molds, resulting in flexible entities called skin patches (SPs) (see Fig. 1 on robot). Every SC features a set of multimodal tactile sensors on the front side and a local controller on the back side. Each SC can locally convert, pre-process, package and forward sensor signals. Neighboring SCs are connected through flexible 4-wire data and power links. The bidirectional cell-2-cell communication allows to organize an arbitrary network of SCs and interconnection of SPs. At least one boundary port of the SC network has to be connected to a computer interface - more connections can be added on demand. Keeping certain data (bandwidth, worst case delay) and power (voltage drop) network limitations in mind, it is possible to serialize a high number of SPs - e.g. to easily equip robots with skin. In this paper, we utilize 3 of the 4 modalities: (i) a tri-axial accelerometer for the open-loop self-organization of SCs on the robot; (ii) a proximity sensor for the detection of approaching objects and contact; (iii) three normal force cells to detect and control contact forces. Currently set to $250 \mathrm{~Hz}$, the update rate of the utilized touch sensors is higher than the $200 \mathrm{~Hz}$ control loop of the robot.

\section{B. Robot}

Our approach is independent of a specific robot, but does not yet support complex actuation mechanisms, beyond common rotatory degrees of freedom (DoFs). The requirements for the control interface are: (i) to publish the number of rotatory degrees of freedom; (ii) to accept (emulated) velocity control values and (iii) to give position feedback. In order to minimize control delays, we utilize the second on board computer (i686, 1.6 GHz, 2 cores, 3 MB L2, 32 GB RAM, Ubuntu 10.04) of HRP-2, to locally process all tactile data. The primary computer executes the $200 \mathrm{~Hz}$ real-time control loop of the stack of tasks (SoT). A stable central body part, like the torso of a humanoid robot or the platform of a mobile robot, is required during self-organization, making it the reference of actions for the motion primitives. With a humanoid robot like HRP-2, a stable balancing controller is thus required. This is no constraint, as our algorithm currently only takes a subset of the available actuators/degrees of freedom (DoFs) into account - namely those related to both arms. The HRP-2 controller generates actuator commands by resolving, in real-time, a set of prioritized tasks. In our experiments, equilibrium is achieved by fixing feet and center of mass position to a static position. Redundancy then allows HRP-2 to realize whole-body manipulation while satisfying the equilibrium tasks. To generate grasping motions with the robot upper-body, a low-priority task is added to the SoT, enforcing both arm velocities.

\section{Self-Organization}

In this section, we describe how open-loop motions and accelerometer readings enable a quickly self-organizing skin.

\section{A. Structural Self-Exploration}

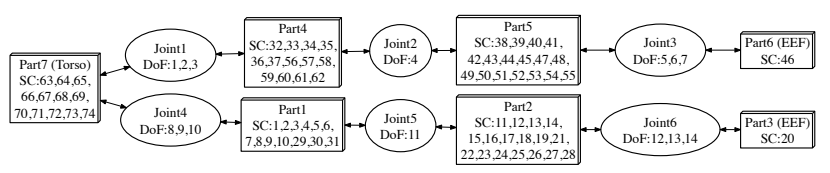

Fig. 4. Structural exploration result: kinematic tree HRP-2's upper body, visualizing the dependencies of joints (featuring one or multiple DoFs) and body parts (featuring one or multiple SCs) towards the torso (root of tree)

The structural self-exploration is an algorithm to automatically discriminate the robot's kinematic tree as a sequence of joints and body parts (see Fig. 3). Most importantly, the 
algorithm quickly discriminates which body parts the SCs have been placed on. We utilize the structural information to suppress cross-coupling effects (e.g. between left and right arm) along the kinematic chain, which is extremely important to avoid unrelated motions in the sensory-motor map - e.g. for tactile guidance. As explained in [10], we measure the normalized gravity vectors $\vec{g}_{s d p}$ of all SCs (s) in an initial pose (p) before (b) and after (a) changing the position of one DoF (d) after the other by $\varphi_{\triangle}$. Both values are compared and if the distance between both normalized vectors is above a pre-defined limit $\left(l_{\text {th }}\right)$, the according entry (being default false) in the binary activity matrix (AM) is set true:

$$
a m_{s d p}=\left|\frac{\vec{g}_{s d p}^{b}}{\left|\vec{g}_{s d p}^{b}\right|}-\frac{\vec{g}_{s d p}^{a}}{\left|\vec{g}_{s d p}^{a}\right|}\right|>l_{\mathrm{th}}, \quad a m_{s d p} \in\{0,1\}
$$

With quasi-static measurements, the unknown robot dynamics can not interfere, but changes in the gravity vector can only be detected if the rotating DoF axis is not primarily aligned with the gravity vector itself. In [10], we provide a solution to this problem. Here, we only perform one (position) incremental run, followed by one decremental run on all DoFs, combining entries from different runs (p) with an element wise 'or'. This simplified approach works, as long as no actuator axis directly attached to the torso, is perfectly aligned with the gravity vector. With a valid activity matrix (a lower triangular form ensures that there is at least one sensor per body part and exactly one stationary reference part), sensor cells with the same activity vectors are body parts, while actuators with the same activity vectors are joints. In the reduced activity matrix (body parts and joints) there is always a pair of body part activity vectors that only differs by a single entry, being the joint connecting both.

\section{B. Sensory-Motor-Map}

The sensory-motor map is a set of matrices, relating SC linear velocities and DoF angular velocities like an inverted jacobian matrix. Each matrix is explored in a pose (p) of the robot and valid around the same. Currently, we explore one matrix of the map per key pose. Each matrix directly maps tactile stimulations into motor velocity vectors, e.g. via a proportional controller, decreasing or increasing tactile stimulation of a SC by motions grounded on the torso (see section $\mathrm{V}-\mathrm{A}$ ). A pose (p) is explored by playing a single velocity sine wave on one DoF (d) after the other, while sensing the generated accelerations with each SC (s) tri-axial accelerometer. All DoF positions are stored to memorize the pose (p) the matrix was explored in. The matrix entries are computed by a weight formula, here along the surface normal $(\mathrm{z})$, relating the maximum deflection of the three accelerometer axes $\left(A_{x}, A_{y}, A_{z}\right)$ and the polarity sign $\left(s_{s, d, p}\right)$ :

$$
w_{s, d, p}^{z}=s_{s, d, p}^{z} \cdot \frac{A_{s, d, p}^{z}}{A_{s, d, p}^{x}+A_{s, d, p}^{y}+A_{s, d, p}^{z}}
$$

We then fuse the structural exploration and the sensorymotor map, by an element wise multiplication of matrix elements (o) between the activity matrix (AM) and the sensory motor map matrix $\left(W_{p}\right)$ of the current pose $(\mathrm{p})$ :

$$
W_{p, \text { new }}=A M \circ W_{p}
$$

Absolutely small SC reaction vectors $\left(\vec{w}_{s, p}^{z}\right)$ need to be cut, as those motions can not be grounded on the torso, but require e.g. locomotion. If left unbalanced, the reaction of SCs at the end of the kinematic chain would be stronger. It is such necessary to normalize each SC vector.

\section{TACTILE TEACHING}

In this section, we explain how we transfer knowledge from human to robot through direct tactile interaction.

\section{A. Tactile Guidance}

Tactile guidance is a direct evasive reaction of body parts on multi-modal tactile stimulation, with the purpose to follow the motion of a teacher. Utilizing simultaneous or sequential contacts, the robot can be driven into different meaningful configurations - here the key poses. We currently provide two different modes: (i) force guidance; (ii) proximity guidance. Force guidance takes the force modality into account and thus requires physical contact with the robot and a sufficiently high force to safely detect the stimulus from background noise. With the pre-contact sensor, and thus proximity guidance, the robot will start to react before the teacher touches the robot (here $\sim 5 \mathrm{~cm}$ before). We utilize the same low-level reaction controllers as for grasping objects.

\section{B. Key Poses}

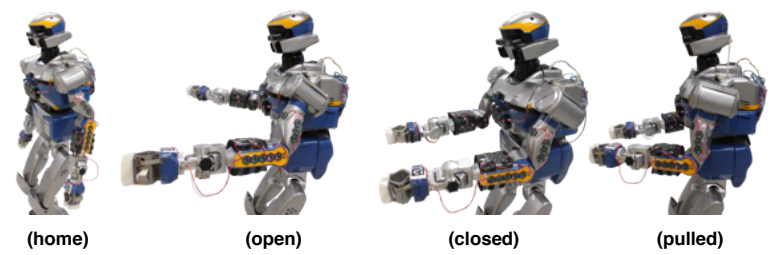

Fig. 5. Key poses are taught to the robot via tactile guidance and serve for the generation of grasping trajectories.

Tactile guidance is utilized to interactively drive the robot into different key poses (see Fig. 5). The robot starts from a 'home' key pose, which we store to be able to return to a safe initial configuration. In the 'open' key pose, both arms are opened widely to make space for an object in between. The 'closed' key pose brings both arms together so an object is between must be made contact with. In the 'pulled' key pose both arms are still together, but the arms are pulled closer to the chest, so any object between the arms necessarily comes into contact with the chest. All key poses are added to the sensory-motor map and serve for grasp trajectory generation.

\section{Touch Areas}

Tactile sensing allows to define areas of special interest - the touch areas (see Fig. 6). For example, we activate the grasping sequence by touching the robot in a 'pat' area (PA) (see Fig. 10). Teaching touch areas is done by selecting 


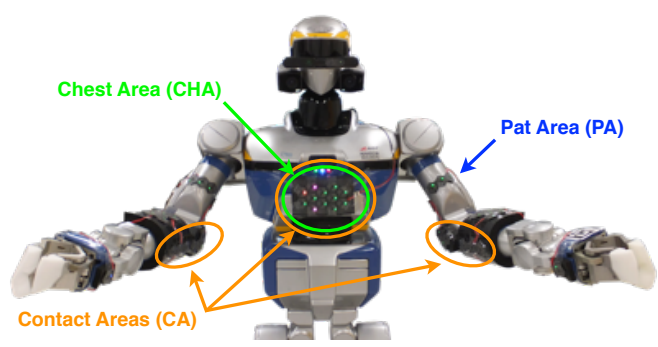

Fig. 6. Touch areas, allow the generation of special tactile events and a differentiation of touch reactions with specialised parameter sets.

a label, activating the attention of the robot (e.g. pushing a button), brushing over the desired area and deactivating attention. While paying attention, the robot evaluates the incoming event stream for new (close) contact events and stores the related unit IDs in a binary vector. For the grasping approach, the operator needs to define the expected contact areas (CA), while remaining IDs are automatically allocated to the non-contact area (NCA). Both areas are allocated different reaction primitives and their events lead to different state changes while grasping objects. The chest area (CHA) serves as a third explicit contact point, besides the left and right arm, which is necessary for a globally stable grasp.

\section{CONTROL STRATEGIES}

In this section, we describe the low and high level control.

\section{A. Tactile Reaction Primitives}

The direct sense of touch allows to implement meaningful direct reactions on tactile stimulation. Here, we instantiate one multi-modal reaction controller for every SC (s), of which all parameters, like gains $\left(P_{m}\right)$ and thresholds $\left(t_{m}\right)$ (refer to Table II), are tunable by the high level statemachine. We compute a proportional value for each sensor modality above a threshold - in this paper only for the three normal force and one proximity sensors $(M=3+1)$. We then calculate desired velocity vectors from the accumulated cell reactions, via the related sensory-motor map vectors $\left(\vec{w}_{s, p}\right)$. Super-imposing the resulting velocity vectors from all SCs, leads to a global robot reaction $\left(\vec{\omega}_{r e}\right)$, which incorporates all sensors:

$$
\vec{\omega}_{r e}=\sum_{s=1}^{S}\left(\vec{w}_{s, p} \cdot \sum_{m=1}^{M}\left(\rho_{m}>t_{m}\right) \cdot\left(\rho_{m}-t_{m}\right) \cdot P_{m}\right)
$$

It is e.g. possible to counteract a slight, large-area precontact reaction, by a strong point force. Modalities can be inhibited or promoted by setting the gain, while the threshold determines the activation level and is very important to suppress the influence of sensor noise. We currently directly act on incoming data, which results in potentially steep velocity responses, but little delay and computational efforts.

\section{B. Postural Trajectory Generation}

The trajectory generation calculates (MATLAB notation for element and boolean operators) velocity commands to transition the robot from a current $\left(\vec{\varphi}_{\text {cur }}\right)$ to a desired $\left(\vec{\varphi}_{\text {des }}\right)$ pose in joint space - e.g. to transition between key poses:

$$
\vec{\omega}_{t r}=\frac{\omega_{\max }\left(\vec{\varphi}_{\text {des }}-\vec{\varphi}_{\text {cur }}\right) .^{*}\left(a b s\left(\vec{\varphi}_{\text {des }}-\vec{\varphi}_{\text {cur }}\right)>\varphi_{a c c}\right)}{\max \left(a b s\left(\vec{\varphi}_{\text {des }}-\vec{\varphi}_{\text {cur }}\right)\right)}
$$

Tunable control parameters define the maximum desired joint velocity $\left(\omega_{\max }\right)$, the desired postural accuracy $\left(\varphi_{a c c}\right)$, a hash name of the pose and a flag if the postural control should be deactivated once the accuracy range was reached. Reaching a desired pose, the motion stops and an event, containing the hash, is emitted. For the overall reaction of the robot, the velocity vectors $\vec{\omega}_{r e}$ and $\vec{\omega}_{t r}$ are super-imposed.

\section{Tactile Events}

TABLE I

Heuristic Tactile Event Levels

\begin{tabular}{|ll||ll|}
\hline \multicolumn{2}{|c||}{ Force Cells } & \multicolumn{2}{c|}{ Pre-contact Sensor } \\
\hline pain force & 0.45 & close contact & 0.80 \\
high force & 0.30 & low proximity & 0.10 \\
medium force & 0.10 & medium proximity & 0.02 \\
low force & 0.04 & high proximity & 0.01 \\
no force & & no proximity & \\
\hline
\end{tabular}

In order to reduce the computational overhead with a growing number of SCs and high update rate, we pre-process tactile signals into events. This is currently done on the computer, as we still wish to log all experimental data. HEX-o-SKIN allows to shift a controllable event generation onto the SCs, extracting information at the earliest stage. This feature will dramatically reduce the average networking and processing load, as most skin areas are not or in constant contact. All high level algorithms already make use of abstract tactile events. Here, we utilize force and proximity events, with a coarse separation into heuristically pre-defined levels (refer to Table I). A new tactile event is emitted on changes between those levels, with a small hysteresis to prevent sensor noise repetitively triggering the same event. Low-level controllers, like the tactile guidance, have to request the full data stream on demand.

\section{$D$. Grasp State Machine}

The whole grasping sequence is split into multiple states (see Fig. 7). On entry, each state sends a set of control directives to the low-level controllers. State changes are triggered by completion events from the low-level controllers, tactile events or user commands. Each state is also assigned a transition to cancel the grasp, which exits the superstate 'execute grasp' and drives the robot into a safe mode. By experience (and two burnt motors) the safest action is not to stop all upper body motions. We now consider the 'open' pose and slow evasion of all pre-contacts to be best. States desiring to interact with an object (e.g. the approach, contact, load or pull state), fail if the desired key pose can be reached without a satisfactory object interaction. In the 'approach' state, the object for example needs to come close to the expected contact area (CA), while forces have to be applicable in the 'load' state. In general, the tactile reaction 


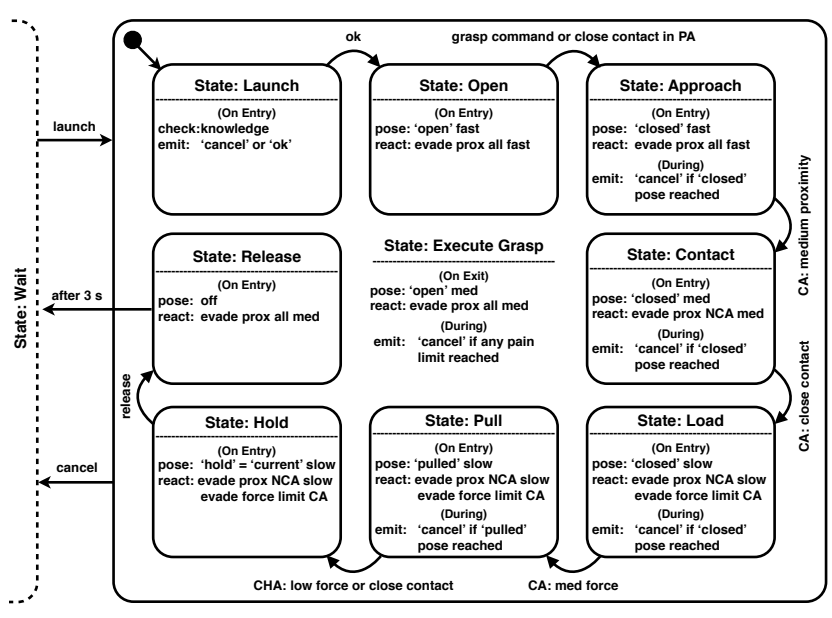

Fig. 7. Control state-machine of the grasping sequence. Trigger events or high level commands transition between discrete grasping states. Entry or exit actions send new parameters to the low-level postural trajectory or tactile reaction controllers. Being in a state activates the conversion of different tactile/proprioceptive events into trigger events.

and the trajectory generation speed become the slower, the closer the robot and object interaction are (refer to Table II). Here, we specifically make use of the pre-contact modality to increase the speed in the approach and contact phase (see Fig. 10). Purely relying on force sensors, a quasi-rigid robot could not interact with a potentially rigid object at high speeds. Forces would ramp up quicker than the reaction time of the robot (due to delays), damaging the robot or the object. There is only three way's out of this dilemma: (i) to add soft compliance to the robot body; (ii) to minimize control delays; (iii) to add further ranging sensor modalities. With HRP-2 and HEX-o-SKIN we utilized: (i) the on-board computer to minimize delays; (ii) a foam layer between the robot and the skin to provide (sensor) hysteresis free compliance; and (iii) pre-contact sensors to slow down motion before contact.

\section{EXPERIMENTS}

In this section, we explain results from our autonomous self-organization algorithms to first grasping experiments.

\section{A. Structural Exploration}

74 SCs have been distributed on the upper body of HRP-2 (see Fig. 4), while having control on 14 actuators (DoFs) of the left and right arm. All SC gravity vectors were measured before and $500 \mathrm{~ms}$ after (to attenuate vibrations) each postural change by $\varphi_{\Delta}=0.1 \mathrm{rad}$. We sampled each vector with an averaging window of $1.0 \mathrm{~s}$ length. The total exploration lasts approximately 70 seconds. A binarizing threshold of $l_{\text {th }}=0.01 \mathrm{~g}$, which is $10 \%$ of the maximum value of $0.10 \mathrm{~g}$, proved to be sensitive enough, but robust against sensor noise and balancing motions of the robot. We could not detect any failure with all $(\mathrm{N} \approx 10)$ conducted runs.

\section{B. Sensory-Motor Map \& Tactile Guidance}

The effectiveness of tactile reactions, and their transfer to motor actions through the sensory-motor map, can be best
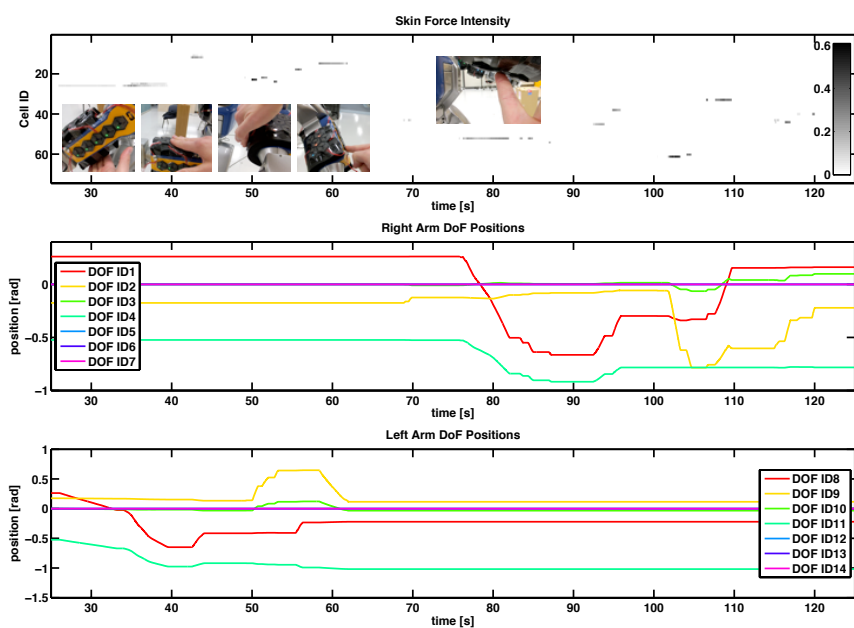

Fig. 8. Force guidance - Stimulations are directly mapped to evasive motor reactions via the sensory-motor map. The first graph shows the force stimulation intensity (grayscale value, white is sub-threshold) over the SC ID and time. The two other graphs show the resulting position of both arms.

evaluated on tactile guidance. Fig. 8 shows a plot of force guidance with both arms, first left then right. The activation threshold of 0.05 force cell readings, approximately relates to $0.6 \mathrm{~N}$, the chosen force gain is 1.0. A single force cell reading of $\rho_{F 1}=0.14$, relating to a force of $1.0 \mathrm{~N}$, leads to commanded velocity of $\vec{\omega}_{r e}=0.09 \mathrm{rad} / \mathrm{s}$ on a single $\mathrm{DoF}-$ which is approximately what can be seen in Fig. 8 between $75 \mathrm{~s}$ and $85 \mathrm{~s}$ with DoF ID1 (neglecting ID4 and 2) and SC ID52. All key poses in Fig. 5 have been taught without touching the robot, via the pre-contact sensor. As the sensorymotor map builds on the fly, it operates as an extrapolation of the closest explored pose - starting from the initial home key pose (see Fig. 5). Due to the lack of the two shear sensing directions on the current SC version, the rotation of some DoFs require a postural change first - which is unintuitive.

\section{Grasping of Unknown Objects}

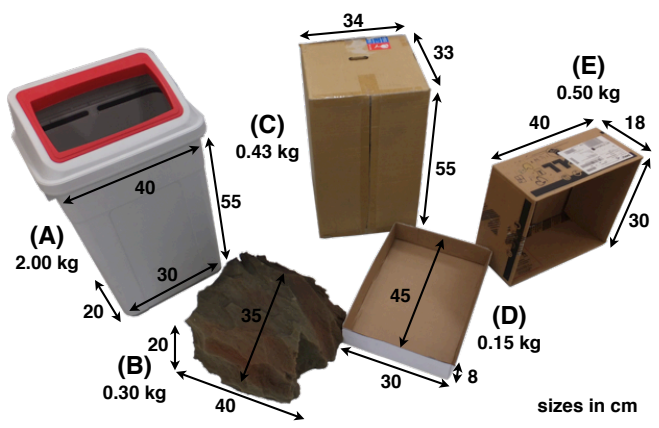

Fig. 9. Objects utilized to test the graping approach: (A) plastic trash bin; (B) sponge rock; (C) moving box; (D) lid of a paper box; (E) computer delivery box. The objects have different weights, shape, hardness and size.

Fig. 9 shows a set of 5 objects with different weight, size, shape and compliance, which we successfully tested our approach on (see Fig. 1). We applied the same set of heuristic parameters (refer to Table II) for all objects. A grasp succeeds, when the robot is able to make contact with the 
TABLE II

EXPERIMENT GRASPING PARAMETERS

\begin{tabular}{|c|c|c|c|c|c|c|c|}
\hline \multirow[b]{2}{*}{ State } & \multicolumn{2}{|c|}{ Force } & \multicolumn{2}{|c|}{ Pre-Contact } & \multicolumn{3}{|c|}{ Pose } \\
\hline & $t_{\mathrm{F}}$ & $P_{\mathrm{F}}$ & $t_{\mathrm{P}}$ & $P_{\mathrm{P}}$ & hash & $\omega_{\max }$ & $\varphi_{\text {acc }}$ \\
\hline F-guide & 0.05 & 1.0 & - & 0.0 & - & - & - \\
\hline open & - & 0.0 & 0.01 & 0.4 & open & 0.4 & 0.01 \\
\hline approach & - & 0.0 & 0.01 & 0.4 & closed & 0.4 & 0.01 \\
\hline contact & - & 0.0 & $\begin{array}{r}-\mathrm{N} \\
0.01 \mathrm{C}\end{array}$ & $\begin{array}{l}0.0 \mathrm{~N} \\
0.4 \mathrm{C}\end{array}$ & closed & 0.1 & 0.01 \\
\hline load & $\begin{array}{l}-\mathrm{N} \\
-\mathrm{C}\end{array}$ & $\begin{array}{l}0.00 \mathrm{~N} \\
0.00 \mathrm{C}\end{array}$ & $\begin{array}{r}0.01 \mathrm{~N} \\
-\mathrm{C}\end{array}$ & $\begin{array}{l}0.01 \mathrm{~N} \\
0.00 \mathrm{C}\end{array}$ & closed & 0.05 & 0.01 \\
\hline pull & $\begin{array}{r}-\mathrm{N} \\
0.10 \mathrm{C}\end{array}$ & $\begin{array}{l}0.00 \mathrm{~N} \\
0.80 \mathrm{C}\end{array}$ & $\begin{array}{r}0.01 \mathrm{~N} \\
-\mathrm{C}\end{array}$ & $\begin{array}{l}0.01 \mathrm{~N} \\
0.00 \mathrm{C}\end{array}$ & pulled & 0.05 & 0.01 \\
\hline hold & $\begin{array}{r}-\mathrm{N} \\
0.10 \mathrm{C}\end{array}$ & $\begin{array}{l}0.00 \mathrm{~N} \\
0.80 \mathrm{C}\end{array}$ & $\begin{array}{r}0.01 \mathrm{~N} \\
-\mathrm{C}\end{array}$ & $\begin{array}{l}0.01 \mathrm{~N} \\
0.00 \mathrm{C}\end{array}$ & - & - & - \\
\hline release & - & 0.0 & 0.01 & 0.2 & - & - & - \\
\hline
\end{tabular}

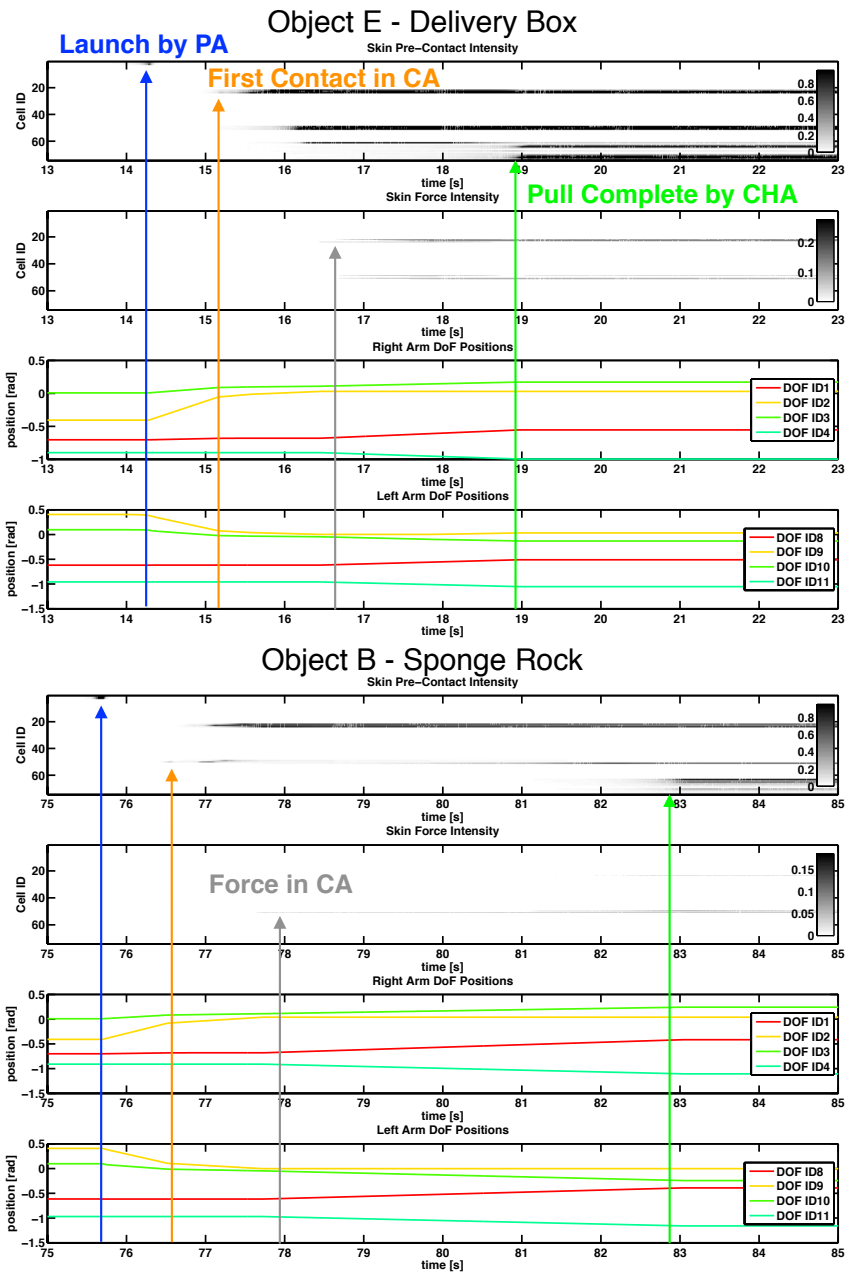

Fig. 10. Proprioceptive and tactile feedback while grasping two objects (E/B) with different compliance (hard/soft) and shape (regular/irregular).

object, apply forces on it and pull it to the chest (see Fig. 10). The robot infers that the graspable object is in between both arms when receiving the initial command. If there is no object, it is to small, too big or can not be pulled, the robot automatically cancels the grasp. With big objects, like $\mathrm{A}$ and $\mathrm{C}$, this case is likely, as contacts on the insensitive wrist disturb the expected sensory feedback. Alas, we could not equip the wrist of the robot with skin sensors due to mechanical constraints. The plastic cover after the wrist does not support force and is such a NCA. We wish to emphasize that no object has been damaged during all experiments. To demonstrate our trust in the system, we let the robot grasp human multiple times (first author). The advantages of the multi-modal approach can be clearly seen in Fig. 10. The precontact modality allows to speed up motions prior to contact and robustly detects when the object touches the chest, which is sufficient to prevent the rotation of objects. But only the force sensor is able to detect and regulate the contact forces.

\section{CONCLUSION}

In this paper, we presented a general tactile approach to grasp unknown objects with a (position controlled) humanoid robot. We demonstrated that a (imprecise) self-explored kinematic model and knowledge transfered by tactile interaction is sufficient.

Acknowledgment: The work on HRP-2 was supported by a 3 month research visit to CNRS-AIST JRL (Joint Robotics Laboratory), UMI3218/CRT, Tsukuba, Japan. Many thanks also to Pierre Gergondet for helping to set up HRP-2.

\section{REFERENCES}

[1] N. Vahrenkamp, M. Przybylski, T. Asfour, and R. Dillmann, "Bimanual grasp planning," 11th IEEE-RAS International Conference on Humanoid Robots, pp. 493-499, 2011.

[2] J. M. Romano, K. Hsiao, G. Niemeyer, S. Chitta, and K. J. Kuchenbecker, "Human-inspired robotic grasp control with tactile sensing," IEEE Transactions on Robotics, vol. 27, no. 6, pp. 1067-1079, December 2011.

[3] K. Hsiao, P. Nangeroni, M. Huber, A. Saxena, and A. Y. Ng, "Reactive grasping using optical proximity sensors," IEEE International Conference on Robotics and Automation, pp. 2098-2105, 2009.

[4] R. Platt, A. H. Fagg, and R. A. Grupen, "Extending fingertip grasping to whole body grasping," IEEE International Conference on Robotics and Automation, pp. 2677-2682, 2003.

[5] A. D. Luca, A. Albu-Schaeffer, S. Haddadin, and G. Hirzinger, "Collision detection and safe reaction with the dlr-iii lightweight manipulator arm," IEEE International Conference on Intelligent Robots and Systems, pp. 1623-1630, 2006.

[6] T. Mukai, S. Hirano, M. Yoshida, H. Nakashima, S. Guo, and Y. Hayakawa, "Whole-body contact manipulation using tactile information for the nursing-care assistant robot riba," International Conference on Intelligent Robots and Systems, pp. 2445-2451, 2011.

[7] Y. Ohmura and Y. Kuniyoshi, "Humanoid robot which can lift a $30 \mathrm{~kg}$ box by whole body contact and tactile feedback," International Conference on Intelligent Robots and Systems, pp. 1136-1141, december 2007.

[8] P. Mittendorfer and G. Cheng, "Integrating discrete force cells into multi-modal artificial skin," IEEE International Conference on $\mathrm{Hu}$ manoid Robots, pp. 847-852, 2012.

[9] —_ "Humanoid multi-modal tactile sensing modules," IEEE Transactions on Robotics, vol. 27, no. 3, pp. 401-410, June 2011

[10] — - "Open-loop self-calibration of articulated robots with artificial skins," IEEE International Conference on Robotics and Automation, pp. 4539-4545, May 2012.

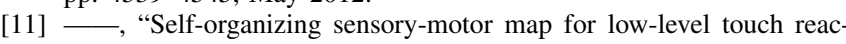
tions," 11th IEEE-RAS International Conference on Humanoid Robots, pp. 59-66, October 2011.

[12] K. Kaneko, F. Kanehiro, S. Kajita, H. Hirukawa, T. Kawasaki, M. Hirata, K. Akachi, and T. Isozumi, "Humanoid robot hrp-2," IEEE International Conference on Robotics and Automation, pp. 1083-1090, April 2004.

[13] N. Mansard, O. Stasse, P. Evrard, and A. Kheddar, "A versatile generalized inverted kinematics implementation for collaborative humanoid robots: The stack of tasks," International Conference on Advanced Robotics, pp. 1-6, 2009. 